
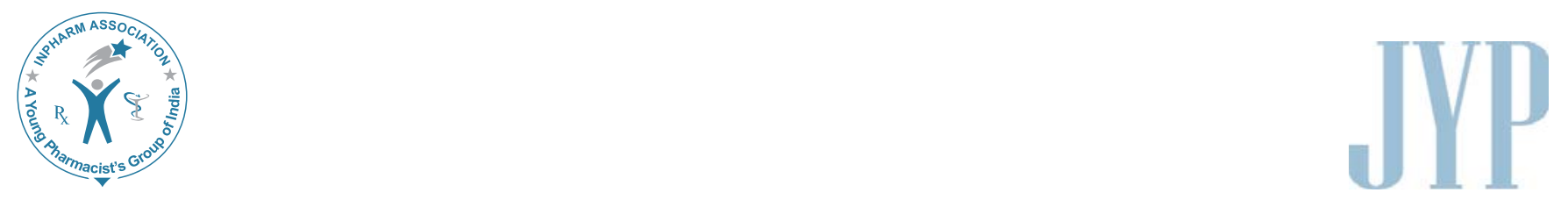

\title{
Efficacy of Extrinsic Stain Removal by Novel Dentifrice Containing Papain and Bromelain Extracts
}

\author{
Chakravarthy PK, Acharya S \\ Department of Public Health Dentistry, Manipal College of Dental Sciences, Manipal University, \\ Manipal, India \\ Address for correspondence: Dr. P. Kalyana Chakravarthy,E-mail: drkalyan81@gmail.com
}

\begin{abstract}
To evaluate the stain removal efficacy of a novel dentifrice containing papain and bromelain extracts (Glodent) in comparison with a control dentifrice (Colgate regular). This was a randomized, positive-controlled, doubleblinded, clinical study. Subjects were randomly divided into one of the two study groups. Pre-treatment and post treatment photographs of the 4 anterior teeth were recorded under standardized conditions and analyzed for lightness or luminosity values using Adobe Photoshop. The difference between the mean pre-treatment luminosity of test and control groups was not statistically significant. In both test and control groups, the post-treatment luminosity was significantly higher than pre- treatment luminosity $(P<0.001$ and $P=0.003$ respectively). The mean post-treatment luminosity for test group was found to be significantly higher than control group. The mean percentage removal of stains for test group was significantly higher than control group. The test dentifrice showed significant stain removal when compared to control which could be attributed to the role of proteolytic enzymes in the test dentifrice.
\end{abstract}

Key words: Bromelain, extrinsic stains, image analysis, papain, proteolytic enzymes, stain removal

\section{INTRODUCTION}

Teeth color is a combination of intrinsic color of the teeth and the presence of extrinsic stains that might accumulate on the tooth surface. ${ }^{[1,2]}$ Extrinsic stains are linked with the adsorption of materials into the acquired pellicle on the enamel surface. ${ }^{[3]}$ Factors influencing extrinsic stain formation include poor tooth brushing technique, smoking,

\begin{tabular}{|l|l|}
\hline \multicolumn{2}{|c|}{ Access this article online } \\
\hline Quick Response Code: & \\
\hline & Website: \\
\hline & www.jyoungpharm.in \\
& DOI: \\
\hline
\end{tabular}

areca nut chewing, dietary intake of colored foods (e.g. red wine, coffee and tea consumption), subject's age and the use of certain cationic agents such as chlorhexidine or metal salts like tin and iron. ${ }^{[1,3,4]}$

Consumers and patients have always had a strong desire for white teeth which has given rise to a growing trend in the increased use of 'over-the-counter' tooth whitening products. Manufacturers of oral care products are constantly developing new approaches for tooth whitening in order to meet the expectations of patients and consumers. Thus, today there is a huge range of products and technologies available that are self-applied and require no professional involvement. ${ }^{[5]}$

The majority of these products work in two possible 
ways. It can be by bleaching of the teeth, or by addition of specific abrasives or chemical agents to toothpaste for the removal and control of extrinsic stain. Abrasives have been shown to effectively remove extrinsic stains along with food debris and plaque and also help in preventing extrinsic teeth stains from reforming. ${ }^{[5]}$

Toothpaste abrasion of dental hard tissues is an important factor in terms of its trade off with cleaning efficacy during the formulation of whitening toothpastes. Hence whitening toothpastes contain additional chemical agents which augment the abrasive cleaning by aiding the removal and/or prevention of extrinsic stains. Many substances like surfactants, peroxides, enzymes, citrate, pyrophosphates and hexametaphosphate were studied previously for their stain removal efficacy. ${ }^{\left[{ }^{[]}\right.}$

Since extrinsic stains are primarily incorporated into the pellicle, it is possible that enzymes such as proteases could help degrade the stained films and potentiate their removal. Early clinical evidence demonstrated that a highly proteolytic mixture of enzymes of fungal origin formulated into toothpaste were effective at reducing extrinsic stain levels as compared to a negative control toothpaste after 6-months of use. ${ }^{[6]}$ Clinical studies have demonstrated the stain removal efficacy of dentifrice containing papain, alumina and sodium citrate. ${ }^{[7-9]}$ Recently an in vitro study reported that a papain and bromelain (proteolytic enzymes) containing dentifrice was more effective in removing stains than the control dentifrice. ${ }^{[10]}$

The methods used to measure extrinsic stain levels in clinical studies include subjective clinician determinations based on indices and objective instrumental methods. The objective instrumental methods used in the past were Vita shade guides, ${ }^{[11]}$ colorimeters, ${ }^{[12]}$ and image analysis of digital photographs of teeth. ${ }^{[13]}$ Digital image analysis can be utilized for assessing stains and overcomes some of the problems associated with some of the subjective and instrumental approaches. ${ }^{[13]}$

Hence the present study aimed to evaluate the stain removal efficacy of a novel commercially available dentifrice containing papain and bromelain (Glodent, Group Pharmaceuticals Ltd, Mumbai, India) in comparison with a control dentifrice (Colgate Regular, Colgate Palmolive India Ltd, Mumbai, India) using a customized digital image analysis system.

\section{MATERIALS AND METHODS}

Subjects and study design

This study was randomized and double blinded (participants and investigators) who compared the novel dentifrice containing papain and bromelain (Glodent) with control dentifrice (Colgate regular). The composition of test dentifrice was Papain, Bromelain, Miswak, Neem and 1000 ppm fluoride and while that of control was calcium carbonate, sorbitol, titanium dioxide, sodium silicate, sodium saccharin and 1000 ppm fluoride dentifrice. Papain and Bromelain are proteolytic enzymes derived from Papaya (Carica papaya) and Pineapple (Ananas comosus).

Before the start of the study, the protocol was approved by the Institute Ethics Committee of Manipal University, Manipal and subjects gave informed consent prior to their participation. For inclusion in the study, the subjects were required to have visible stains on maxillary incisors with Lobene score of $>1$; at least 4 maxillary anterior teeth without restorations; no oral prostheses; no untreated caries. Lobene stain index (1968) involves assessment of extent and intensity of stain on the gingival and body regions of the labial surface of incisor teeth. Intensity scores were: 0- no stain, 1- light stain, 2- moderate stain, 3- heavy stain. Extent scores were: 0 - no stain, 1 - stain covering up to $1 / 3^{\text {rd }}$ of the region, 2 - stain covering $>1 / 3^{\text {rd }}$ to $2 / 3^{\text {rd }}$ of the region and 3 - stain covering $>2 / 3^{\text {rd }}$ of the region. Many stain indices proposed recently were mainly to determine the efficacy of anti-stain agents, or to investigate the interaction between chlorhexidine and dietary constituents. Subjects were excluded from participating in the study if there were generalized recession of the gingiva, or generalized malocclusion, or overlapping/spacing of anterior teeth, inability to comply with brushing instructions (e.g,dexterity or comprehension issues), obvious periodontal disease, or facial calculus on the anterior teeth. A total of 100 subjects were screened in out-reach centers of Manipal College of Dental Sciences, Manipal out of whom 29 subjects satisfied the inclusion criteria. Out of these 24 subjects consented to participate in the study. This study was conducted for 2-months (November-December 2010).

Eligible subjects were randomly assigned to one of the two treatment groups and called to record the baseline photographs of their 4 maxillary anterior teeth. After briefing the purpose of the study to the participants, oral hygiene and tooth brushing instructions were given. Subjects who were divided into two groups were provided with the assigned products (either of the toothpaste and Colgate classic toothbrush with soft bristles) and were required to brush in front of a mirror for 2 minutes twice a day, covering the entire toothbrush head with the dentifrice each time. Test and control dentifrices tubes were painted in white color and delivered by a person not involved in examination. All investigators and participants 
were unaware of the identity of the tubes given. Tubes were identified as either the "Group 1" or the "Group 2 " tubes. Identity of each tube was revealed only after 2-weeks when the subjects were recalled for the follow-up examination. During this visit, standardized photographs of the 4 maxillary anterior teeth were repeated. Subjects were given a printed timetable for 2 -weeks and were asked to check at the respective area after tooth brushing to ensure the compliance of brushing frequency. At the end of 2-weeks, participants were offered free treatment for scaling and polishing [Figure 1].

Photographs of the teeth were captured in a customized wooden box with standardized lighting conditions, a chin rest and high resolution digital camera mounted on a platform.

\section{Wooden box}

A customized wooden box was made with plywood in dimensions of $18 \times 18 \times 18$ inches. One end of the box was kept open for the subject to keep the head and the other end for the recorder to capture the image of the area of interest. The open ends of the box were covered with black drapes suspended with closed coiled springs at the upper and lower margins of the box [Figure 2]. These drapes had a slit opening for the subjects to place their head inside the box, for the investigator to check for positioning of the head, focusing, and adjustments needed for image capture. The inner surface of the box was painted matt black to prevent reflection of light [Figure 3]. For the box to be portable, a handle was fitted on the top. The floor of the box was mounted with a rigid customized chin cup fabricated with acrylic on one end and a rigid acrylic platform for mounting the digital camera on the other end. Both these units had provisions for adjusting height.

\section{Lighting conditions}

To avoid variation in lighting and possibly affect the stain intensity measurement, a standard light source was kept on the floor between the chin rest and the platform for the camera. The light was artificially powered with operational specifications of 220-240V, 5W power and 6500K. (Phillips India Ltd., India).

\section{Camera}

Digital images were captured using a high resolution camera (DSC - S2000, 10.1 megapixels Sony ${ }^{\circledR}$ India,) fitted inside the wooden box in front of the patient's chin rest on a

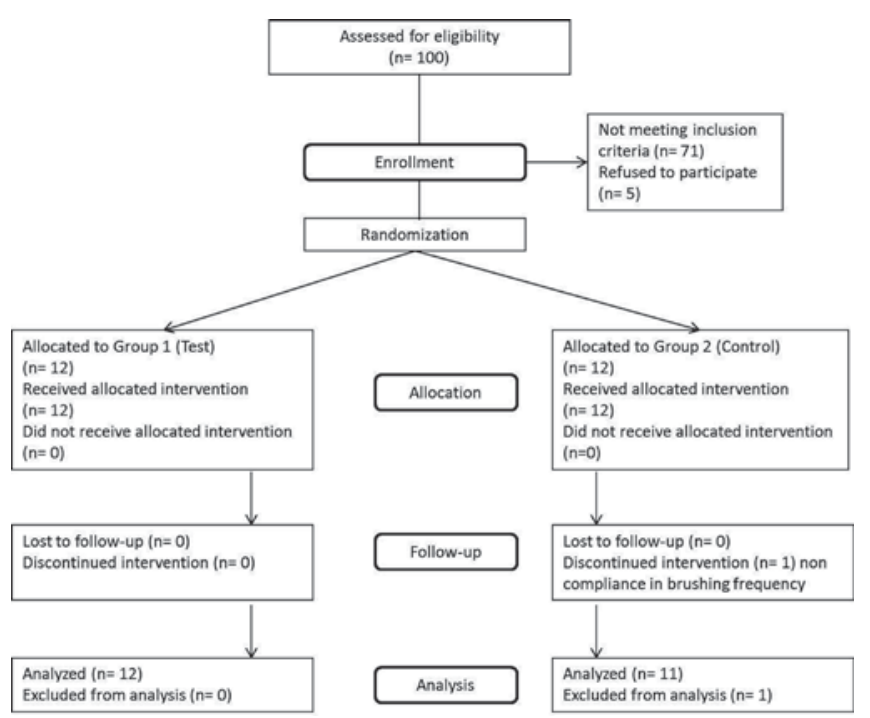

Figure 1: Consort flow chart showing the various steps in the study

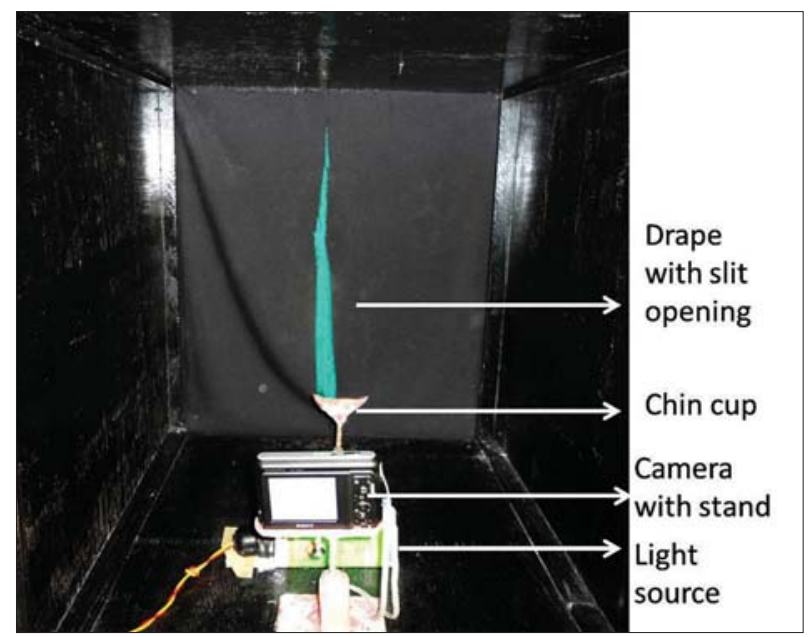

Figure 2: Inside view of the box

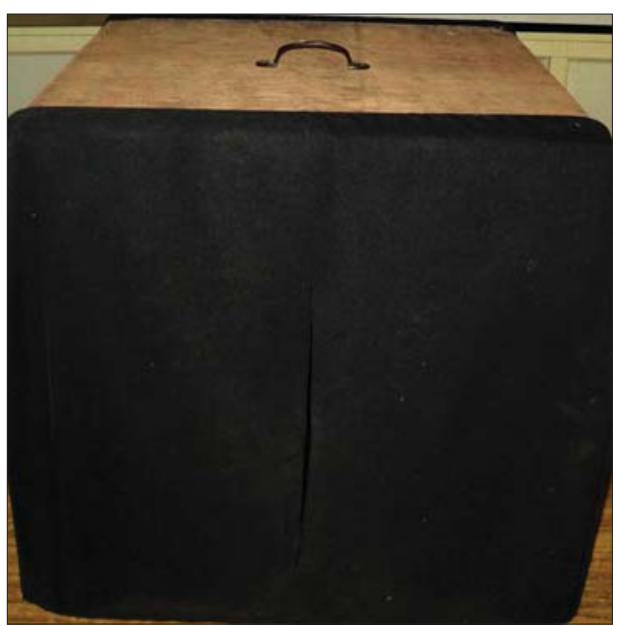

Figure 3: Outside view of the box

platform with fixed base. Images were captured without flash and were transferred to a computer. 


\section{Image capture}

Before capturing the images all the subjects were instructed to place their head inside the wooden box and place the chin on the chin rest in order to capture the image of their 4 anterior teeth. Cheek retractor was used to visualize the teeth during photography. After focusing on the area of interest, the digital image was captured. Subjects were asked not to move during the image capture.

\section{Scoring}

The images were opened in Adobe Photoshop (version 8.0) and the area of interest was outlined using a magnetic lasso tool in the toolbar option of Adobe Photoshop software. Once the outline was selected, mean L-value of all the pixels within the area of interest was shown in the histogram window provided in the software. ${ }^{[10]}$ The amount of stain was graded digitally using only the Luminosity or Lightness ( $\mathrm{L}$ value of Adobe Photoshop version 8.0. $\mathrm{L}$ that represents the lightness of the stain, with $\mathrm{L}=255$ being white, and $\mathrm{L}=0$ being black). This procedure was repeated for 5 subjects randomly to check the reliability in obtaining the $L$ values. The coefficient of reliability (Pearson correlation) of the image analysis system was found to be 0.98 .

Statistical analysis

The Lightness values were entered in SPSS version 14 (SPSS Inc., Chicago, IL, USA). Independent sample t-test was performed to compare the pre-treatment Luminosity, post-treatment Luminosity and percent removal of stains. Paired $t$-test was used to compare the pre-treatment and post-treatment Luminosity between the test and control groups. A $P$-value of 0.05 was considered statistically significant. Percentage removal stains was calculated by [(Post-treatment Luminosity - Pre-treatment Luminosity)/ Pre-treatment Luminosity] $\times 100$.

\section{RESULTS}

The mean age of the study population was $32.09 \pm 5.05$ with a range of 22-40 years. Out of 24 participants, 23 completed the follow-up (12 in Test and 11 in Control). The difference between the mean pre-treatment Luminosity of test $(157.95 \pm 16.93)$ and control groups (143.82 \pm 17.48) was not statistically significant $(P=0.062)$. In both test and control groups, the post-treatment Luminosity was significantly higher than pre-treatment Luminosity $(P<0.001$ and $P=0.003$ respectively). The mean posttreatment Luminosity for test group $(170.76 \pm 14.14)$ was found to be significantly higher than control group (147.81 \pm 16.47) $(P=0.002)$ [Table 1]. The mean percentage removal of stains for test group $(8.44 \pm 4.83)$ was significantly higher than control group $(2.92 \pm 2.89)(P=$ 0.004) [Table 2 and Figure 4].

\section{DISCUSSION}

The current study evaluated the clinical stain removal efficacy of a novel dentifrice containing papain and bromelain in comparison with a control dentifrice. In this study an economical and portable customized digital image analysis system was developed and used to evaluate stain removal.

A number of studies have reported the use of clinical indices for stain evaluation. These indices are quick and easy to use, but they face reliability issues because of their subjective nature. To overcome such problems, image analysis has been used as an objective method in the past to assess stain build up in vitro on acrylic and enamel slabs. ${ }^{[1,15]}$ It was also shown to be an effective tool for assessing plaque ${ }^{[16]}$ and stains ${ }^{[13]}$ clinically. However, the methodology used in this study was different although the underlying construct for color assessment was same.

Image analysis is very sensitive even for minor changes in stain removal. This might facilitate in reduction of the

Table 1: Comparison of luminosity values among test and control groups

\begin{tabular}{|c|c|c|c|c|}
\hline Group & $\mathbf{N}$ & $\begin{array}{c}\text { Pre-treatment } \\
\text { luminosity } \\
\text { Mean } \pm \text { SD }\end{array}$ & $\begin{array}{c}\text { Post-treatment } \\
\text { luminosity } \\
\text { Mean } \pm \text { SD }\end{array}$ & $P$-value \\
\hline Test group (Glodent) & 12 & $157.95 \pm 16.93$ & $170.76 \pm 14.14$ & $<0.001^{\dagger}$ \\
\hline $\begin{array}{l}\text { Control Group (Colgate } \\
\text { regular) }\end{array}$ & 11 & $143.82 \pm 17.48$ & $147.81 \pm 16.47$ & $0.003^{\dagger}$ \\
\hline$P$-value & & $0.062^{\ddagger}$ & $0.002^{\ddagger}$ & \\
\hline
\end{tabular}

${ }^{\dagger} P$-value for paired $t$-test, ${ }^{\dagger} P$-value for independent sample $t$-test

Table 2: Mean percent removal of stains among test and control groups

\begin{tabular}{lccc}
\hline $\begin{array}{l}\text { Percent } \\
\text { removal of } \\
\text { stains }\end{array}$ & $\begin{array}{l}\text { Test group } \\
\text { (Glodent) } \\
\text { Mean } \pm \text { SD }\end{array}$ & $\begin{array}{c}\text { Control Group } \\
\text { (Colgate regular) } \\
\text { Mean } \pm \text { SD }\end{array}$ & $P$-value \\
\hline & $8.44 \pm 4.83$ & $2.92 \pm 2.89$ & 0.004 \\
\hline
\end{tabular}

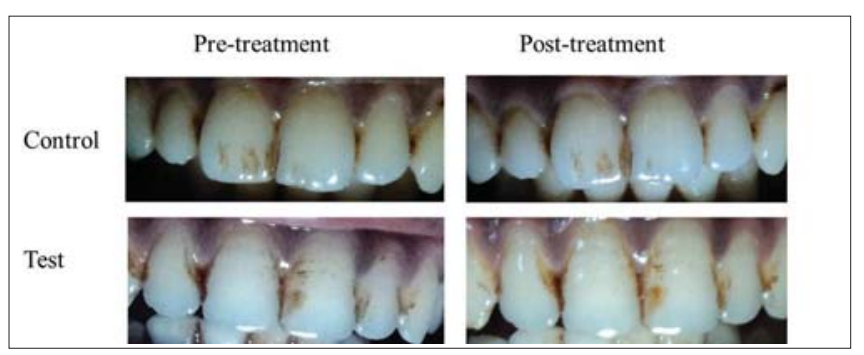

Figure 4: Pre-treatment and post-treatment photographs of subjects 
duration of the clinical trials involving stain removal to as short as 2 -weeks. This short duration of the study can reduce the costs of conducting a clinical trial, improve patient compliance, reduce attrition of subjects while eliminating subjective bias. The apparatus developed for our study was light in weight and could easily be carried to different field situations and could be replicated elsewhere.

The stain removal efficacy of novel dentifrice was assessed in terms of Lightness values only. The mean pre-treatment Lightness value for test and control group did not differ significantly. But the mean post-treatment Lightness value was significantly higher for test than control dentifrice. Similar results were reported by a previous in-vitro study ${ }^{[10]}$ using this test dentifrice. Clinical studies using toothpaste containing a mixture of papain, alumina and sodium citrate (Rembrandt) have also reported a significant stain removal. ${ }^{[7-9]}$ The test dentifrice in the present study contained extracts of papain and bromelain, which are proteolytic enzymes. They disrupt and / or remove the protein portion of the pellicle/ plaque layer that forms on the surface of teeth over time, thus removing the stains that are bound to these proteins. The control dentifrice was chosen as Colgate regular since the relative dentine abrasive value was nearly the same as test dentifrice [67 (test) and 70 (control) respectively]. Hence, significant increase in the mean Lightness values for the test dentifrice group could partly be attributed to these enzymes.

Limitations of the study were small sample size and habits like tobacco and areca nut usage was not accounted. Habits like tobacco and areca nut usage can have a direct effect on staining and are widely prevalent in this area. Although our study did not account for habits, the result remains valid in terms of maintenance of stain removal on a daily usage of the dentifrice whilst using these stain causing agents. In this particular geographic area where chewing areca nut is culturally acceptable, it is less likely to find subjects not having habits and but had stains on their teeth. Hence we could not warrant that subjects have not used these substances during the study period. Also, the shelf life of test dentifrice might be shorter than usual dentifrices because of papain and bromelain being proteolytic in nature. Overall, our study can be considered as a pilot study to evaluate the stain removal of this novel dentifrice and a feasibility study of digital image analysis in the assessment of stains. The results of this study can be useful to plan the needed clinical trials with large number of participants.

\section{REFERENCES}

1. Watts A, Addy M. Tooth discoloration and staining: A review of the literature. Br Dent J 2001;190:309-16.

2. Joiner A. Tooth color: A review of the literature. J Dent 2004;32(Suppl 1):3-12.

3. Joiner A, Jones NM, Raven SJ. Investigation of factors influencing stain formation utilizing an in situ model.Adv Dent Res 1995;9:471-6.

4. Nathoo S. The chemistry and mechanisms of extrinsic and intrinsic discoloration. J Am Dent Assoc 1997;128:6S-10S.

5. Joiner A. Whitening toothpastes: A review of the literature. J Dent 2010;38s:e17-24.

6. Harrison JWE, Salisbury GB, Abbott DD. Effect of enzyme toothpastes upon oral hygiene. J Periodontol 1963;34:334-7.

7. Lyon TC, Parker WA, Barnes GP. Evaluation of effects of application of a citroxain-containing dentifrice. J Esthet Dent 1991;3:51-3.

8. Emling RC, Levin S, Shi X, Weinberg S, Yankell SL. Rembrandt toothpaste stain prevention with and without the use of Peridex. J Clin Dent 1992;3:59-65.

9. Emling RC, Shi X, Yankell SL. Rembrandt toothpaste: Stain removal following the use of Peridex. J Clin Dent 1992;3:66-9.

10. Kalyana P, Shashidhar A, Meghashyam B, SreeVidya KR, Sweta S. Stain removal efficacy of a novel dentifrice containing papain and bromelain extracts-in vitro study. Int J Dent Hyg 2011;9:229-33.

11. Kakar A, Rustogi K, Zhang YP, Petrone ME, De Vizio W, Proskin HM. A clinical investigation of the tooth whitening efficacy of a new hydrogen peroxide-containing dentifrice. J Clin Dent 2004;15:41-5.

12. Walsh TF, Rawlinson A, Wildgoose D, Marlow I, Haywood J, Ward JM. Clinical evaluation of the stain removing ability of a whitening dentifrice and stain controlling system. J Dent 2005;33:413-8.

13. Collins LZ, Naeeni M, Platten SM. Instant tooth whitening from a silica toothpaste containing blue covarine. J Dent 2008;36:S21-5.

14. Lath DL, Johnson C, Smith RN, Brook AH. Measurement of stain removal in vitro: a comparison of two instrumental methods. Int J Dent Hyg 2006;4:129-32.

15. Lath DL, Smith RN, Guan YH, Karmo M, Rook AH. Measurement of stain on extracted teeth using spectrophotometry and digital image analysis. Int J Dent Hyg 2007;5:174-9.

16. Smith RN, Brook AH, Elcock C. The quantification of dental plaque using an image analysis system: Reliability and validation. J Clin Periodontol 2001;28:1158-62.

How to cite this article: Chakravarthy PK, Acharya S. Efficacy of extrinsic stain removal by novel dentifrice containing papain and bromelain extracts. J Young Pharmacists 2012;4:245-9.

Source of Support: Nil, Conflict of Interest: None declared. 